\title{
Solder sealing method for paraffin-filled microcavities
}

\author{
Hugo Nguyen, Johan Bejhed, Johan Köhler and Greger Thornell \\ The Ångström Space Technology Centre, Department of Engineering Sciences, Uppsala \\ University, Sweden \\ E-mail: hugo.nguyen@angstrom.uu.se
}

\begin{abstract}
.
Demonstrated and investigated here is a method to seal microfluidic systems by soldering. As a particularly difficult case of growing importance, the sealing of openings contaminated with paraffin wax was studied. Solder paste, screen printed on a metallized silicon, substrate was melted locally through application of 6.5 to $10 \mathrm{~V}$ to a $5 \Omega$ copper film resistor for a few seconds and found able to drive an intermediate layer of paraffin away and seal a $0.2 \mathrm{~mm}$ diameter circular via by wetting to a surrounding copper pad. Although verified to be robust, the process did result in failing seals on excessive heating because of consumption of the pads. Correctly performed, the technique provided a seal at least withstanding a pressure of 8 bar for $8 \mathrm{~h}$ at $85^{\circ} \mathrm{C}$.
\end{abstract}

Key words: seal, sealing, solder, soldering, micro-, fluidic, paraffin

\section{Introduction}

The closure of fluidic microsystems is not a simple task, although if significant dead volumes or bulky sealings are allowed, the task is easier. As the systems' small dimensions, and the efficiency demands of microtechnology, seldom allow neither for individual, mechanical adjustments nor complex assemblies with gaskets and such, the sealing method must rely on a reproducible interfacial geometry or use an individually adaptable or forgiving sealing mechanism.

A particularly demanding case is the sealing of paraffin cavities - a challenge of growing concern as this substance has been used extensively for microactuation [1-12] for instance in valves and pumps. Paraffin waxes exhibit a low surface energy and tend to wet most solids very well. As a result the surroundings of a cavity opening are easily contaminated with either a rough solid, or a slippery liquid, layer making both physical and chemical sealing difficult. Most bulk micromachined paraffin-based actuators have therefore been sealed, after filling them with melted paraffin, by gluing a cover glass over the opening and a significant area around it, or by dispensing a fair amount of epoxy glue over it [1-4]. For prototyping and moderately miniaturized components this is satisfactory, but besides efficiency (as with batch processing) and quality, process compatibility and the prospect of integration will suffer. Paraffin-filled actuators with paraffin being in direct contact with a fluid actuated [5-7], or those with cavities or diaphragms created by deposition of elastic material on a defined volume and shape of paraffin in solid state [8-12], often termed as surface micromachined paraffin actuators, do not, of course, need any plugging.

In this paper, a sealing method for paraffin-filled cavities in microsystem based on screen-print soldering is proposed and evaluated. Especially this method's ability to handle contamination and the resulting strength of the sealing are investigated.

\section{Device design}

The above mentioned bulk micromachined actuators [1-4] contain a paraffin reservoir accessed via a channel ending with an opening at the device surface. The design of the samples for experiments in this work mimics the inlets of these devices. The samples are made of single crystalline silicon, are $5.5 \times 8.5 \times 0.3 \mathrm{~mm}$ in size, and contain a centred $0.2 \mathrm{~mm}$ diameter via widening into a square funnel embraced by a meandering copper heater between two handle-shaped pads for electrical connection, Figure 1(a). The side of the sample chip with the funnel and the electrodes represents the exterior of a paraffin container, and the recessed opening is to facilitate in-plane sealing. 
Above the funnel, but not as to cover the circular via, a clover-shaped solder pad is deposited by screen printing through the stencil mask depicted in Figure 1(b). The circular disk preventing solder paste from obstructing the via is suspended by four spokes. The thickness of $0.1 \mathrm{~mm}$ of the mask determines the thickness of the solder paste layer.

(a)

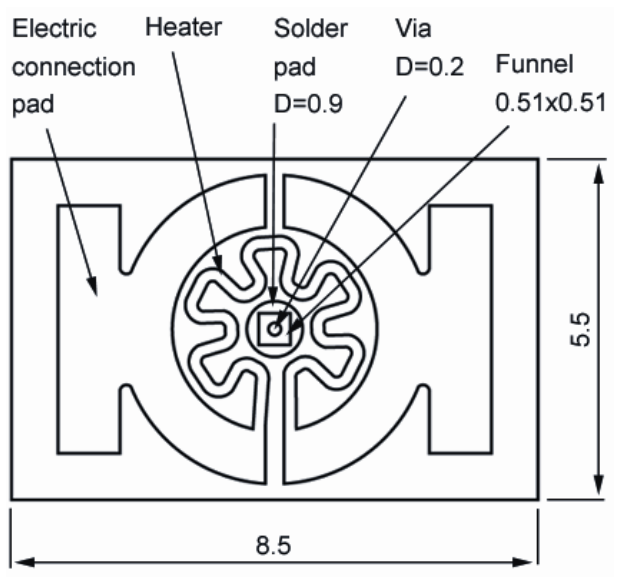

(b)

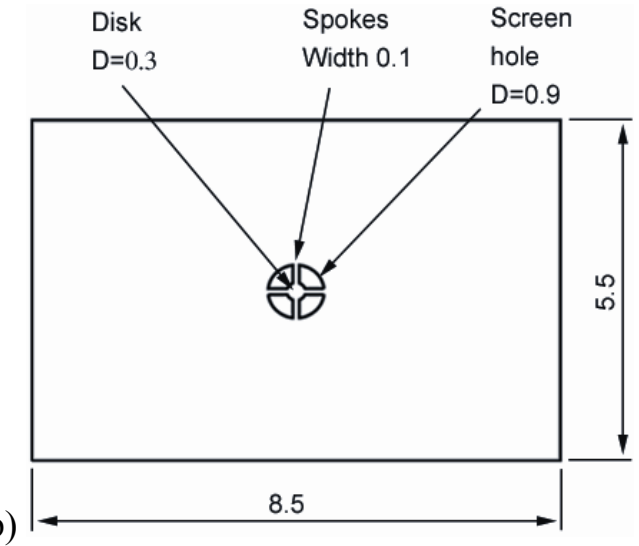

Figure 1. Design of the sample with its solder pad and combined heater and connection pad, (a), and design of the stencil mask for solder paste printing (b). (All dimensions are in $\mathrm{mm}$, D stands for diameter.)

\section{Methods and materials}

\subsection{Device fabrication and sample preparation}

The samples were made in-house, whereas the stencil masks - made of stainless steel - were obtained from a commercial supplier (HP Etch, Sweden).

A standard (100) silicon wafer with a thickness of $300 \mu \mathrm{m}$ was used for fabrication of the samples. Oxide masking and standard lithography followed by potassium hydroxide $(\mathrm{KOH})$ etching on time to a depth of $130 \mu \mathrm{m}$ was used to create the funnel, whereas the circular via was made by means of aluminium masking and patterning, followed by standard BOSCH Deep Reactive Ion Etching (DRIE) from the backside after having protected the other side with a sputtered layer of aluminium. After metal and oxide stripping, a $500 \mathrm{~nm}$ thick oxide was grown, and the heater structure was deposited by evaporation of $30 \mathrm{~nm}$ titanium and $150 \mathrm{~nm}$ copper through a stencil mask (HP Etch, Sweden). Finally, the test chips were diced out.

To create realistic surface conditions on the samples, they were coated with an approximately 20-50 $\mu \mathrm{m}$ thick layer of paraffin (paraffin wax 5405, Ter Hell \& Co GmbH, Germany) with a melting point of $52^{\circ} \mathrm{C}$. Lead-free solder paste (R276LF, Kester) with a melting point of about $150^{\circ} \mathrm{C}$ was printed on top of this through the stencil mask described above, Figure 1(b). This mask was aligned manually under a microscope and removed vertically from the sample after screen printing of the solder paste.

\subsection{Sealing.}

Prior to soldering, the samples were put in thermal contact with a $35 \times 70 \times 4 \mathrm{~mm}$ glass substrate (Borofloat) using a thermally conducting paste with a thickness of about $100 \mu \mathrm{m}$ to ensure similar thermal conditions for all soldering experiments.

The LabView environment (version 7.0, National Instruments) and a PCI card (PCI-1711S, Advantech) regulating a power supply, were used both to control the soldering process, and to register time, and the current and voltage applied to the heaters. The voltage, applied through probes, was ramped from zero until the solder paste melted and was then typically held constant for a few seconds in order for the molten solder to wet the pad. Then, the voltage was ramped down in a controlled way in order not to make ambient conditions dominate the solidification. These phases were designed with respect to the solder provider's general recommendation for the preheating, soaking, and cooling 
phases of the solder. However, the durations of the ramping up and ramping down phases, 15 and $5 \mathrm{~s}$ long, respectively, were much shorter than the recommendation (obviously addressing oven soldering), since, in this case, the heat generated by the copper heater was transferred to the solder paste via the silicon substrate of the sample instead of through convection. A schematic voltage vs. time profile is shown in Figure 2. (A general treatise on soldering is provided in [13].)

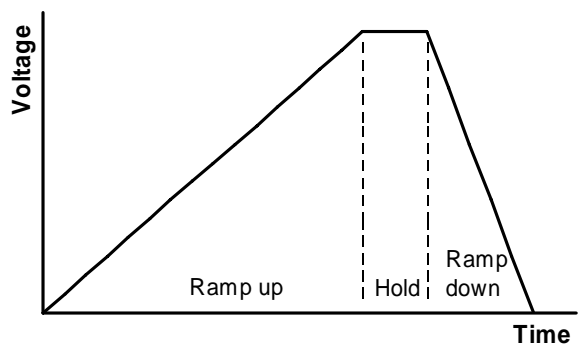

Figure 2. A schematic soldering profile in terms of applied voltage vs. time.

The resistance of the heaters was measured to $5 \pm 0.2 \Omega$ at room temperature (so that the power could be estimated). Prior to the actual test runs, a working reference voltage and holding time were determined in a series of trials. (In this, the time and voltage during the holding phase was varied with increments of $1 \mathrm{~s}$ and $0.5 \mathrm{~V}$, respectively, to the extent where the solder failed to seal the opening.) Based on this, a sensitivity study with four additional voltage-time combinations was carried out. Table 1 contains these combinations with VT denoting the reference setting, and V and T followed by plus and minus, denoting an increase and decrease in holding voltage and holding time, respectively. Sample 3 was powered twice, since a first run with $6 \mathrm{~V}$ applied failed to melt the solder.

Table 1. Holding times and voltages for the seven samples soldered.

\begin{tabular}{cccc}
\hline $\begin{array}{c}\text { Sample } \\
\#\end{array}$ & $\begin{array}{c}\text { Soldering } \\
\text { type }\end{array}$ & $\begin{array}{c}\text { Holding } \\
\text { voltage } \\
(\mathrm{V})\end{array}$ & $\begin{array}{c}\text { Holding } \\
\text { time } \\
(\mathrm{s})\end{array}$ \\
\hline 1A \& 1B & $\mathrm{VT}$ & 8.5 & 1 \\
2 & $\mathrm{~T}-$ & 8.5 & 0 \\
3 & $\mathrm{~V}-$ & 6.5 & 1 \\
4 & $\mathrm{~T}+$ & 8.5 & 5 \\
5A \& 5B & $\mathrm{V}+$ & 10 & 1 \\
\hline
\end{tabular}

\subsection{Evaluation}

The soldering process was monitored through a light microscope, and the quality of the seals was judged primarily from the melting, wetting, and solidifying behaviours observed. This procedure also worked as a screening for subsequent testing.

Two types of leak tests were performed using pressurized nitrogen gas from the backside of the samples as this would be most faithful to the actual case with a pressurized cavity, and would also not assist the plug-shaped sealing by forcing it into the funnel. A volumetric leak test with nitrogen under a pressure of 5 bar for about 10 minutes at room temperature was conducted on samples $1 \mathrm{~A}, 1 \mathrm{~B}, 4,5 \mathrm{~A}$ and $5 \mathrm{~B}$ to verify whether the seal was tight or not. For this purpose a pressure gauge was used to detect pressure drops. Of all samples deemed good, sample 1B was chosen for a flowmetric leak test at $85^{\circ} \mathrm{C}$ with nitrogen under a pressure of 8 bar and for a duration of 8 hours in order to test the sealing's resistance to elevated operating temperature and to detect any slow degradation. A modified flow meter calibrated for jig leakage, and a climate cabinet (Cooled Incubator, ES 250 (HY)) was used. In both test cases, the samples were clamped symmetrically with a tightened o-ring on the pressurized backside and another o-ring on the front side serving as a cushion.

After testing, excess paraffin was removed by gentle rubbing in hot water (about $60^{\circ} \mathrm{C}$ ) followed by immersion in boiling water for half a minute, and short rinsing in acetone and isopropanol. To find the 
primary reason for the variation in quality, the samples were examined by means of light microscopy (Olympus AX70, Japan). Following this, cross sections were prepared by cutting the samples along their main axis, approximately $1 \mathrm{~mm}$ from the funnel, vacuum potting them standing in cylindrical blocks of room-temperature-curing epoxy (EpoFix, Struers), and eventually grinding and polishing these until the centre of the vias were exposed. After a standard $20 \mathrm{~nm}$ thick Au/Pt sputter metallization, the cross sections were investigated by Scanning Electron Microscopy, SEM (Zeiss, DSM 960A).

\section{Results}

Figure 3 shows a sample before screen printing of solder paste, a close-up of a sample with paraffin and solder paste, and a sample where the soldering is completed. The clover-shaped patch of paste in panel (b) is aligned to the solder pad and offers a free passage to the via. The white embankments around the solder seal in (c) is residual paraffin. (In Figure 3(b) the granular surface resulting from solidified paraffin wax is clearly visible.)

(a)

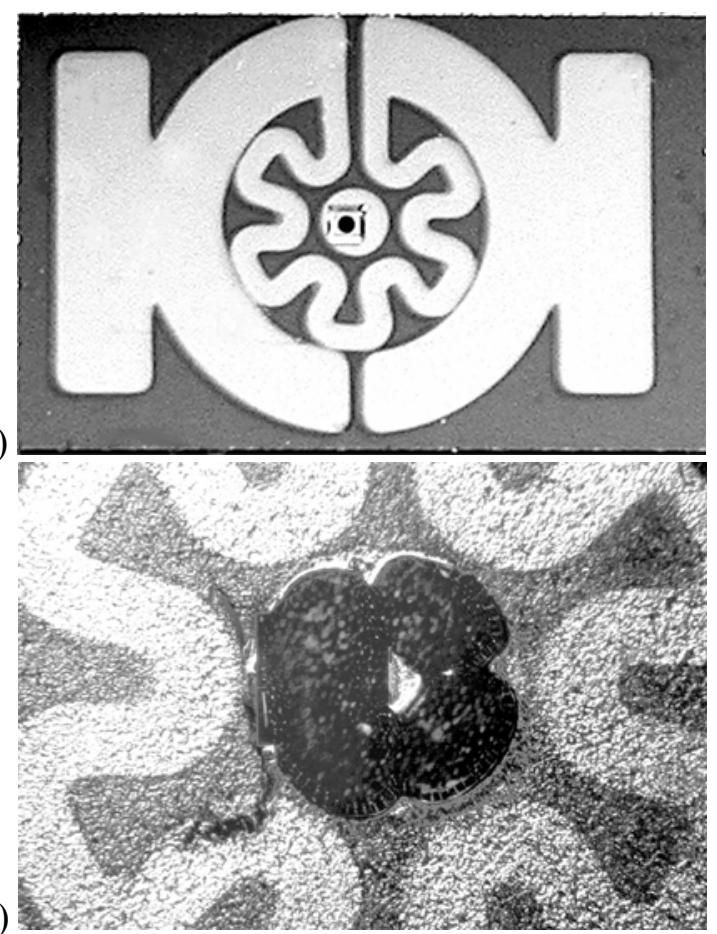

(b)

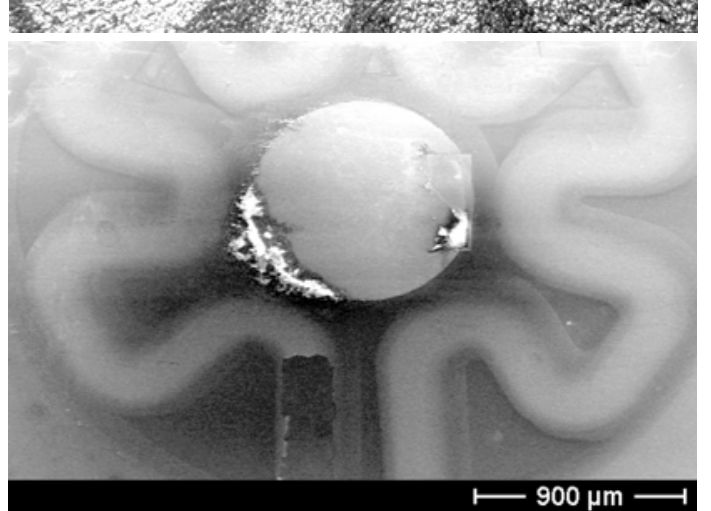

Figure 3. Pictures of a not completed sample, (a), a sample with screen-printed solder paste on its paraffin coated surface, (b), and a sealed sample, (c). (SEM was used for the last picture, light microscopy for the other.)

Figure 4 is a sequence of pictures from a representative ramp up, showing how the paraffin wax successively melts (panel 1-3) partly overlapping with the melting of the solder paste (panel 3) releasing a plume of fusing agent vapour (panel 4). 

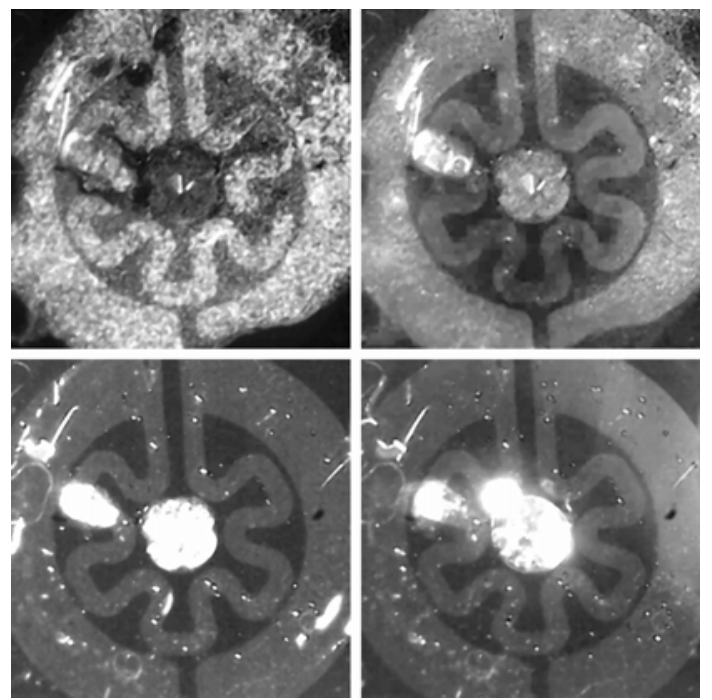

Figure 4 Paraffin wax starting to melt (upper left), continuing its melting (upper right), finishing its melting overlapping with melting of solder paste (lower left), and solder paste releasing its fusing agent as a plume (lower right), in a representative heating phase.

Table 2 presents the results from leak testing together with comments from the inspections during and after processing.

Table 2. Results from leak testing, and comments from inspections. (A dash in the second column indicates that leak testing was not performed. "Positive" refers to a leak-proof sample and vice versa.)

\begin{tabular}{ccc}
\hline Sample & Leak test & Comments from visual inspection \\
\hline 1A \& 1B & positive & good \\
2 & - & good \\
3 & - & good, powered twice, solidified \\
immediately \\
4 & positive & slightly shrunken, copper seems \\
& & consumed \\
shrunken \\
5B & negative & bad, hole through via \\
\hline
\end{tabular}

The flowmetric test at $85^{\circ} \mathrm{C}$ with nitrogen under a pressure of 8 bar on sample $1 \mathrm{~B}$ did not result in any leakage or visible degradation of the seal.

Figure 5 shows SEM pictures of cross-sections from samples 1A and 3. Figure 6 shows a close-up of the typical interface between the solder and the silicon. No trace of the Ti/Cu metallization could be found where solder was present.
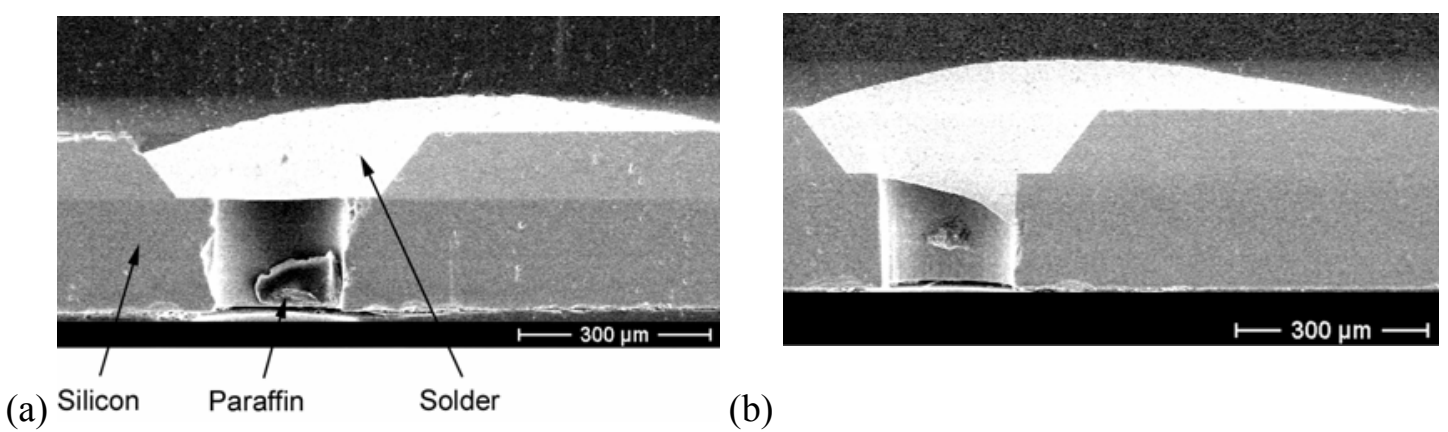

(a) Silicon Paraffin Solder

(b)

Figure 5. Cross-sections of sample 1A and 3, (a) and (b), respectively. The solder seals (bright) lie asymmetrically upon the funnels. 


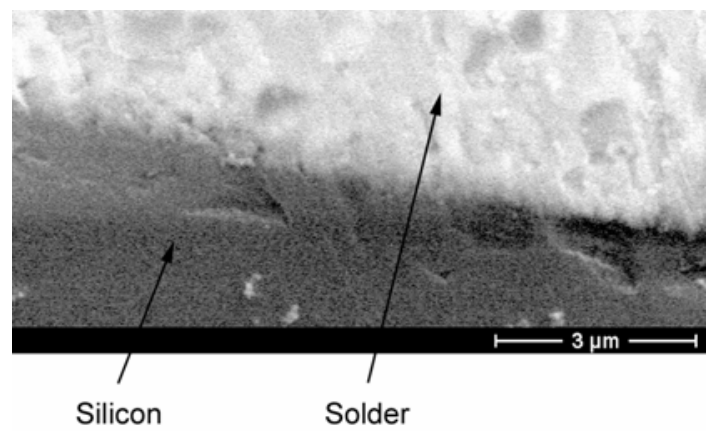

Figure 6. Close-up of typical interface between silicon and solder

Figure 7 shows an SEM cross-section of sample 4 which was subjected to a moderate heating power but for five times as long as any other sample. Note that the profile of the solder seal is significantly higher, and its base shorter than that presented in Figure 5.

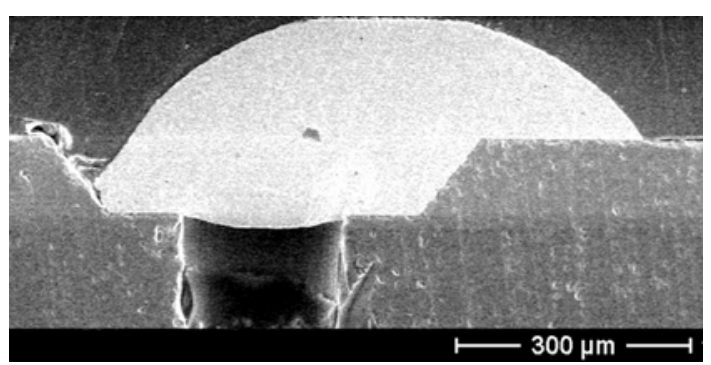

Figure 7. Cross-section of sample 4. Note that the solder does not reach to the top left edge of the funnel.

Figure 8 and 9 show SEM pictures of tilted cross-sections of samples $5 \mathrm{~A}$ and $5 \mathrm{~B}$, respectively. In both cases, the holding time was optimal, but the applied voltage $10 \mathrm{~V}$. (The voids in the epoxy matrix come from air having expanded out from the via during vacuum hardening of the epoxy.)

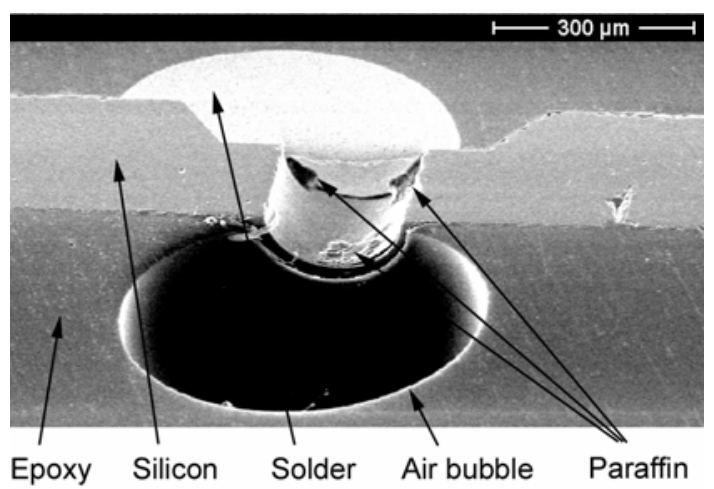

Figure 8. Tilted cross-section of sample 5A.

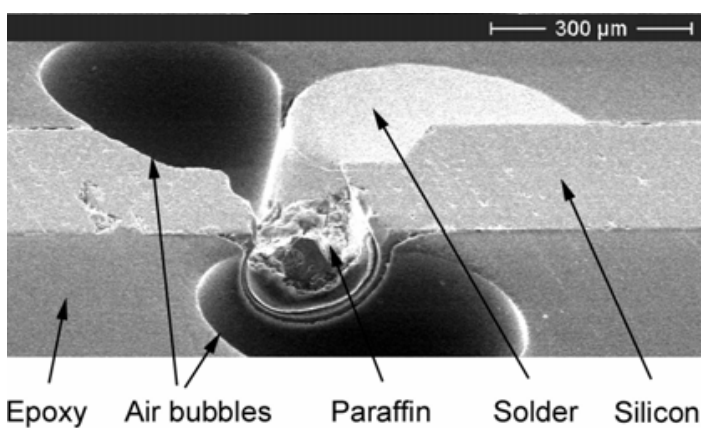

Figure 9. Tilted cross-section of sample 5B with clearly failed seal and a large quantity of residual paraffin present. 
Figure 10 shows the surface quality of the via part of the device.

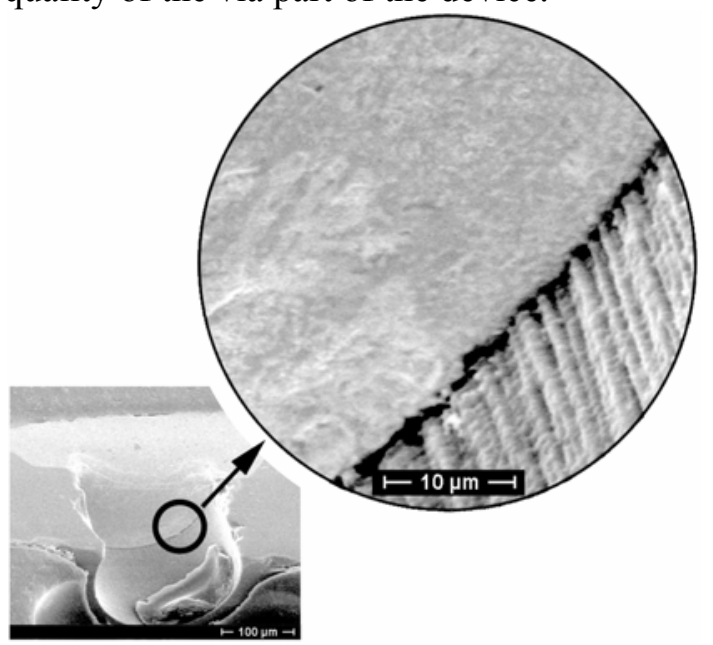

Figure 10. Tilted cross-section of sample 1A with overview and close-up of solder interface where the via meets the funnel showing a vertically sharp, and a horizontally vague texture.

\section{Discussion}

The exact mechanism for the sealing could not be determined from this work. However, it is clear that the solder paste does not penetrate the paraffin wax layer as an entity. Instead, it appears to dissolve in the melted paraffin and gather - perhaps particle by particle - on the underlying pad. Here, it either regains its integrity releasing a plume of pure fusing agent, or a vapour consisting of both fusing agent and residual paraffin. (See Figure 4.)

All the samples presented in this paper had pads for solder paste not perfectly - but sufficiently well aligned to the funnels, Figure 3 (a). This is mainly a result of the larger thermal expansion of the shadow mask than the silicon wafer in the evaporation process, as verified by the fact that the misalignment increased with the distance to the two clamped corners of the mask-and-wafer sandwich. (A feature distortion caused by the mask being slightly arced between these two points was also observed.) It is reasonable to believe that although this is the very reason for an asymmetrically positioned seal as shown for instance in Figure 5, the degree of asymmetry depends on the heating power and time during soldering.

In no case did the solder exhibit poor wetting to its pad. However, on some occasions the good wetting, as demonstrated by the sharp solder angle of samples $1 \mathrm{~A}$ and 3 (Figure 5), was disguised when, as it is believed, a too large quantity of paste had been applied and forced to wet a pad of more or less fixed size. This evidence of wetting together with the lack of paraffin in the interface between solder and silicon dioxide (possibly with metal traces), to the extent confirmable given the resolution and contrast provided in the electron microscopy, show that the molten solder indeed drove the paraffin away from the copper pad and adhered itself to it. Moreover, the leak test at elevated temperature indicates qualitatively that there was at least no paraffin left in form of a radially continuous patch around the via along the sealing interface. If not resulting in disengagement of the solder plug (for instance because of a release of spotwise adhesion or mechanical locking), melting of the paraffin in this test would at least have increased the leak rate.

Regarding the quality of the etched surfaces, cross sections (see Figures 5-9) show that the wet etched funnel part was very smooth whereas the dry etched via was rougher. The close-up in Figure 10 demonstrates a perhaps surprising texture consisting of a sharp vertical ripple stemming from poor discretization of the circles in the template used for lithography. Superimposed on this is the expected (and much vaguer) ripple from the cycle-wise etching of the DRIE process. 
With an exception for sample 3, Figure 5(b), the cross-sections showed that the solder plugs did not penetrate into the vias, but instead - save for a slight bagging - stayed flush with the bottom of the funnel part as had it been an inverted mesa. Although the reason for the exception is not clear, it is easy to understand that the directionality of the evaporation process did not provide enough metal on the vertical sidewalls to produce a solder-wettable surface. Altogether, this not only speaks in favour of an adhesion-governed sealing mechanism, but dismisses mechanical locking of the solder plug, for instance to the sidewall ripple commonly encountered with DRIE - an otherwise likely contributor, had the plug extended far enough. The confinement of the solder to the metal pattern indicates that the method could be used for the sealing of rather densely populated wafers. To which extent neighbouring devices can be individually sealed, however, requires a dedicated investigation of the thermal cross talking.

As for the operational window of the process applied to these devices, $6.5 \mathrm{~V}$ or slightly below but not $6 \mathrm{~V}$ seems to be the lower limit for the applied voltage judging from sample 3 . In this case, the melting actually occurred at the end of the holding phase and was followed by immediate solidification on ramping down, which shows that the lower limit also of the holding time had been reached in this experiment.

Too high power and too long holding time, on the other hand, tend to result in poor quality. The smaller foot prints, and resulting larger curvatures, of the solder bumps of samples $4,5 \mathrm{~A}$ and 5B in Figures 7-9, respectively, indicate less attraction between the solder and the substrate. In fact, on observation of sample 4 during heating, the solder droplet was seen to contract laterally during the long holding phase. Samples 5A and 5B which were sealed with a holding time of $1 \mathrm{~s}$, but a close to $40 \%$ higher power (neglecting the change in resistance with temperature), failed to form a leak-light plug and to cover the via, respectively. It is believed that excessive heating, or alternatively a long holding time, causes all copper of the pad to diffuse into the molten solder terminating the good wetting.

From the successful flowmetric leak test, although its main purpose was to reveal any continuous film of paraffin at the solder-silicon interface, the continuous allowable operating temperature for the seal was found to be at least $85^{\circ} \mathrm{C}$.

It should be mentioned that the selection of materials, and the experimental conditions, were to some extent governed by the use of paraffin actuators in space applications [2,3]. The small dimensions of the samples primarily serve the purpose of getting as many samples as possible out of a silicon wafer.

Finally, a couple of application-related remarks can be made. The design, manufacturing and operation of the seal are straightforward, and should be easy to integrate with most microstructures. To permit stacking of wafers or chips on top of the seal, either further optimization of the funnel size versus solder amount, or an intermediate planarization, must be made as the bumps here protrude above the funnel. Applied to the sealing of a paraffin-based actuator's cavity two important questions remain to be answered: Will the heating be localized enough not to cause a significant drainage of the reservoir and a correspondingly shorter stroke? Is a seal strength of 8 bar as verified here (although it could be much higher) enough for most actuators of this kind - especially since paraffin can deliver considerable expansion even when subjected to megapascal pressures? Clearly, additional investigation is needed to find this out. Meanwhile, one can argue that should these issues prove to be of concern, they can probably be remedied in many applications by sensible design. For instance, a relatively long and narrow channel from the reservoir to the opening will lessen the amount of paraffin subjected to sealing heat, and also allow for paraffin close to the seal to be solid and hence supportive during normal operation of the device. Solder paste with lower melting point, yet above the operation temperature of the actuator, is likely to mitigate the risk of vaporization of the paraffin wax during sealing. 


\section{Conclusions}

A method to seal contaminated openings - such as those resulting from filling of microfluidic devices with paraffin for actuation purposes - by soldering supported by localized heating from application of less than $10 \mathrm{~V}$ to a $5 \Omega$ resistor has been demonstrated. The resulting seals, being less than a millimetre in diameter, have been shown to, at least, endure pressures of 8 bar at a temperature of $85^{\circ} \mathrm{C}$ for 8 hours, which is well above the melting point of the paraffin used here. Cross sectioning and electron microscopy show no traces of paraffin in the sealing interface.

\section{Acknowledgement}

The authors would like to express their gratitude to the European Space Agency, who is the sponsor of this project falling under the Micro/Nano Satellite Technologies project (ESA contract number 11041/04/NL/PM).

\section{References}

[1] Klintberg L, Svedberg M, Nikolajeff F, and Thornell G 2003 Fabrication of a paraffin actuator using hot embossing of polycarbonate Sensors and Actuators A 103 307-16

[2] Klintberg L, Karlsson M, Stenmark L, and Thornell, G 2003 A thermally activated paraffinbased actuator for gas-flow control in a satellite electrical propulsion system Sensors and Actuators A 105 237-46

[3] Klintberg L, Karlsson M, Stenmark L.; Schweitz, J-Å, and Thornell G 2002 A large stroke, high force paraffin phase transition actuator Sensors and Actuators A 96 189-95

[4] Svedberg M, Nikolajeff F, and Thornell G 2006 On the integration of flexible circuit boards with hot embossed thermoplastic structures for actuator purposes Sensors and Actuators A $125534-47$

[5] Pal R, Yang M, Johnson B N, Burke D T and Burns M A 2004 Phase change microvalve for integrated devices Anal. Chem. 76 3740-8

[6] Liu R H, Yang J, Lenigk R, Bonanno J and Grodzinski P 2004 Single-use, thermally actuated paraffin valves for microfluidic applications Sensors Actuators B 98 328-36

[7] Liu R H, Yang J, Lenigk R, Bonanno J and Grodzinski P 2004 Self-contained, fully integrated biochip for sample preparation, polymerase chain reaction amplification, and DNA microarray detection Anal. Chem. 76 1824-31

[8] Klintberg L and Thornell G 2002 A thermal microactuator made by partial impregnation of polyimide with paraffin J. Micromech. Microeng. 12 849-54

[9] Bodén R, Lehto M, Simu U, Thornell G, Hjort K and Schweitz J-Å 2006 A polymeric paraffin actuated high-pressure Sensors and Actuators A 127 88-93

[10] Carlen E T and Mastrangelo C H 1999 Simple, high actuation power, thermally activated paraffin microactuator Transduceers'99 2 1364-67

[11] Carlen E T and Mastrangelo C H 2002 Surface micromachined paraffin-actuated microvalve $J$. Microelectromech. Syst. 11 408-20

[12] Selvaganapathy P, Carlen E T and Mastrangelo C H 2003 Electrothermally actuated inline microfluidic valve Sensors Actuators A 104 275-82

[13] Principles of Soldering and Brazing, G Humpston and D M Jacobson, ASM International, 1993 